\title{
Inroduction on Recent Trends and Perspectives in Fake News Research
}

Fake news, especially on social media, is now viewed as one of the main digital threats to democracy, journalism, and freedom of expression $[1,4,11,15]$. Our economies are not immune to the spread of fake news, either, with fake news being connected to stock market fluctuations and massive trades $[6,14]$. The goal of this special issue is to promote the exchange of research and studies that (1) aim to understand and characterize fake news and its patterns and how it can be differentiated from other similar concepts, such as false/satire news, misinformation, disinformation, among others, which helps deepen our understanding of fake news, and (2) systematically detect fake news by determining its credibility [7], verifying its facts, assessing its writing style [5, 15], or identifying its propagation $[8,13]$. To facilitate further research in fake news, this special issue presents recent research on misinformation and fake news.

Popularity of social media $[3,10]$ and recent events align perfectly to the focus of this special issue as (1) the rise of social media has resulted in wider reachability across geographic regions and different ethnic and socio-economic groups, and (2) we are experiencing an "infodemic" of information with low credibility such as fake news and conspiracies on COVID-19 [2, 9, 12], presenting opportunities for state and individual actors to manipulate news for malicious gains through fake news.

Here, we categorize accepted papers into two types: those that study the media and those that focus on techniques. The media, the main vehicle to spread fake news, has evolved from traditional forms such as newspapers and TV to becoming dominant social networks, such as Facebook, Twitter, and TikTok. Similarly, advances in machine learning and automated methods have led to techniques that can automatically spread fake news through social bots and can adapt to varying conditions and multiply the effect of fake news.

This special issue presents significant findings on the analysis of fake news data and means to spread it. For instance, it shows research language-theoretic fact checking to determine the veracity of claims; reliable expert identification for fact checking and means to check the credibility of the data as well as of the credibility of the social media user; and analysis of fake news sharing through Zika virus spread that analyze threat, severity cues, and the resulting coping cues.

CCS Concepts: • Human-centered computing $\rightarrow$ Collaborative and social computing theory, concepts and paradigms; Empirical studies in collaborative and social computing; • Computing methodologies $\rightarrow$ Natural language processing; Machine learning; • Security and privacy $\rightarrow$ Social aspects of security and privacy; • Applied computing $\rightarrow$ Sociology; Computer forensics;

Additional Key Words and Phrases: Fake news, news verification, disinformation, misinformation, fact-checking, knowledge graph, deception detection, information credibility, social media

ACM Reference format:

Reza Zafarani, Huan Liu, Vir V. Phoha, and Javad Azimi. 2021. Inroduction on Recent Trends and Perspectives in Fake News Research. Digit. Threat.: Res. Pract. 2, 2, Article 13 (March 2021), 3 pages. https://doi.org/10.1145/3448634

(c) 2021 Copyright held by the owner/author(s).

2576-5337/2021/03-ART13

https://doi.org/10.1145/3448634

Digital Threats: Research and Practice, Vol. 2, No. 2, Article 13. Publication date: March 2021. 
While covering both science and applications with a balance between theory and practice, this special issue selects papers based on their scientific merit. Each submission was reviewed by at least three reviewers and went through at least two rounds of reviews. A brief review of the selected papers is given below.

The article "Towards Automated Factchecking: Developing an Annotation Schema and Benchmark for Consistent Automated Claim Detection," which is a collaboration between a non-profit organization and academia, presents methods to verify the veracity of a claim and experience of an in-use system using these methods. The work presents a benchmark methodology consisting of an annotation schema, crowd-sourcing, task definition, and machine learning methods. The claim detection system uses a universal sentence representation using crowd-sourcing human annotators, resulting in a dataset of 5,571 sentences labelled according to the schema and machine learning methods. Their system outperforms the state-of-the-art claim detection systems ClaimBuster and ClaimRank.

"A Perfect Storm: Social Media News, Psychological Biases, and AI" presents a study of information bias by using concepts from psychology and artificial intelligence. The article presents an Information Bias Anchoring Model (IBAM) that explains how uncertainty might result from social media news and leads to information bias and anchoring. The article develops IBAM within the context of explainable artificial intelligence. The article also discusses how technical rationality and individual biases might mitigate uncertainty. Finally, the article discusses how to reduce information bias anchoring.

"Fake News Sharing: An Investigation of Threat and Coping Cues in the Context of Zika Virus" studies how fake news is shared in the healthcare context. It is well known that threat situations and responses necessitate information sharing and seeking among the public. Thus, to study fake news sharing, it would be effective to identify threat and coping related cues as indicators of share-worthiness of fake news in social media. The authors try to answer research questions associated with fake news sharing in the context of Zika virus, e.g., how do threat and coping related cues influence fake news sharing? This study paves the way for the efficient monitoring of viral fake messages.

Finally, "Identifying Real-World Credible Experts in the Financial Domain" addresses an important issue in detecting disinformation. One way of checking whether some information is useless, misleading, or even malicious is to look at whether its author or producer is credible or trustworthy. This article tries to employ this approach in the financial domain by identifying attributes that can distinguish credible users on the Internet who are indeed trustworthy experts in the real world. The authors gather the list of credible financial experts from real-world financial authorities and then analyze the distribution of attributes of about 10K stock-related Twitter users and their 600K tweets over six months in 2015/2016, and over 2.6M typical Twitter users and their 4.8M tweets on November 2nd, 2015, in their attempt to find which attributes are related to real-world expertise.

\section{REFERENCES}

[1] Hunt Allcott and Matthew Gentzkow. 2017. Social media and fake news in the 2016 election. f. Econ. Perspect. 31, 2 (2017), 211-36.

[2] United Nations. 2020. UN tackles "infodemic" of misinformation and cybercrime in COVID-19 crisis. (2020).

[3] Andrew Perrin. 2015. Social networking usage: 2005-2015. Pew Research Center.

[4] David Pogue. 2017. How to stamp out fake news. Sci. Am. 316, 2 (2017), 24-24.

[5] Martin Potthast, Johannes Kiesel, Kevin Reinartz, Janek Bevendorff, and Benno Stein. 2017. A stylometric inquiry into hyperpartisan and fake news. arXiv:1702.05638. Retrieved from https://arxiv.org/abs/1702.05638.

[6] Kenneth Rapoza. 2017. Can "fake news" impact the stock market? Forbes (2017).

[7] Niraj Sitaula, Chilukuri K. Mohan, Jennifer Grygiel, Xinyi Zhou, and Reza Zafarani. 2020. Credibility-based fake news detection. In Disinformation, Misinformation, and Fake News in Social Media. Springer, 163-182.

[8] Soroush Vosoughi, Deb Roy, and Sinan Aral. 2018. The spread of true and false news online. Science 359, 6380 (2018), $1146-1151$.

[9] Chen Yang, Xinyi Zhou, and Reza Zafarani. 2020. CHECKED: Chinese COVID-19 fake news dataset. arXiv:2010.09029. Retrieved from https://arxiv.org/abs/2010.09029.

[10] Reza Zafarani, Mohammad Ali Abbasi, and Huan Liu. 2014. Social Media Mining: An Introduction. Cambridge University Press.

Digital Threats: Research and Practice, Vol. 2, No. 2, Article 13. Publication date: March 2021. 
[11] Reza Zafarani, Xinyi Zhou, Kai Shu, and Huan Liu. 2019. Fake news research: Theories, detection strategies, and open problems. In Proceedings of the 25th ACM SIGKDD International Conference on Knowledge Discovery \& Data Mining. 3207-3208.

[12] Xinyi Zhou, Apurva Mulay, Emilio Ferrara, and Reza Zafarani. 2020. ReCOVery: A multimodal repository for COVID-19 news credibility research. In Proceedings of the 29th ACM International Conference on Information \& Knowledge Management (CIKM'20). Association for Computing Machinery, New York, NY, 3205-3212. DOI : https://doi.org/10.1145/3340531.3412880

[13] Xinyi Zhou and Reza Zafarani. 2019. Network-based fake news detection: A pattern-driven approach. ACM SIGKDD Explor. Newslett. 21, 2 (2019), 48-60.

[14] Xinyi Zhou and Reza Zafarani. 2020. A survey of fake news: Fundamental theories, detection methods, and opportunities. ACM Comput. Surv. 53, 5 (2020), 1-40.

[15] Xinyi Zhou, Reza Zafarani, Kai Shu, and Huan Liu. 2019. Fake news: Fundamental theories, detection strategies and challenges. In Proceedings of the 12th ACM International Conference on Web Search and Data Mining. 836-837.

Reza Zafarani

Huan Liu

Vir V. Phoha

Javad Azimi

Guest Editors 